\title{
Primary alveolar soft part sarcoma of the right femur and primary lymphoma of the left femur: A case report and literature review
}

\author{
YIYUAN CAO, HANFEI ZHANG, YANJUAN QU and MEIYAN LIAO \\ Department of Radiology, Zhongnan Hospital of Wuhan University, Wuhan, Hubei 430071, P.R. China
}

Received January 11, 2015; Accepted September 24, 2015

DOI: $10.3892 / \mathrm{ol} .2015 .3906$

\begin{abstract}
Alveolar soft part sarcoma (ASPS) is a rare soft tissue tumor, particularly in bone, which usually affects young patients. Primary lymphoma of bone (PLB) is a rare form of non-Hodgkin's lymphoma that occurs primarily in bone. In the present report, the case of a 42-year-old woman affected by ASPS and PLB in the left and right femur, respectively, is presented. The radiological features of digital radiography, computed tomography and magnetic resonance imaging examination are discussed, and compared with previous findings reported in the literature. To the best of our knowledge, the present study is the first case of ASPS and PLB occurring concomitantly in a patient.
\end{abstract}

\section{Introduction}

Alveolar soft part sarcoma (ASPS) is a rare soft tissue tumor that accounts for $\sim 0.5-1.0 \%$ of all soft tissue sarcomas (1). ASPS was first described in 1952, and has rarely been reported since (2). ASPS involves several locations, particularly muscles and deep soft tissues of the body (3). In adults, ASPS is often localized in the lower extremities, while the head and neck are the predominant sites for ASPS in children (4). Park et al reported six cases of primary ASPS of bone in 1999, and demonstrated the bone origin of this condition based on their radiological findings (5). To date, the number of published studies reporting cases of ASPS is limited $(3,6,7)$.

Primary lymphoma of bone (PLB) is an extranodal lymphoma that arises from the medullary cavity and manifests as a localized, solitary lesion (8). PLB represents $3 \%$ of all primary malignant bone tumors, and $1 \%$ of all malignant lymphomas (8). First reported by Oberling in 1928 (9), PLB has been described as a malignant, lymphoid infiltrate within

Correspondence to: Professor Meiyan Liao, Department of Radiology, Zhongnan Hospital of Wuhan University, 169 Donghu Road, Wuhan, Hubei 430071, P.R. China

E-mail: 1iao681018@sina.com

Key words: alveolar soft part sarcoma, primary lymphoma of bone, computed tomography, magnetic resonance imaging, femur bone, with or without cortical invasion or soft tissue extension, and without concurrent involvement of regional lymph nodes or distant viscera (10). Non-Hodgkin's lymphoma constitutes the majority of PLBs, and the most common subtype is diffuse large B-cell lymphoma $(11,12)$, while T-cell PLB is rare (13).

Second primary malignances (SPMs) are newly developed malignant neoplasms that present synchronously or metachronously in a patient with a known malignant disease (14). The incidence of SPM is not rare (15-18). However, a limited number of cases of primary intraosseous ASPS and PLB have been reported in the literature thus far. To the best of our knowledge, the present report is the first case of intraosseous ASPS and PLB occurring concomitantly in the same patient. Written informed consent was obtained from the patient.

\section{Case report}

A 42-year-old woman presented to the Department of Radiology of Zhongnan Hospital of Wuhan University (Wuhan, China) with continuous pain in her right hip and occasional pain in her left leg, in addition to dizziness and heart palpitations. The patient had been experiencing these symptoms during the two months prior to the date of admission to the hospital in June 2010.

The results of a blood routine test performed prior to the date of admission suggested anemia, and the patient had been receiving treatment for anemia during one month at her local hospital, but the pain in her right hip worsened. Therefore, the patient was referred to the Zhongnan Hospital of Wuhan University. Physical examination identified a firm mass with a poorly delineated margin that was palpable in the right hip. The left leg examinations did not indicate any abnormalities. The red blood cell count was $2.23 \times 10^{12}$ cells/1, and the levels of hemoglobin were $66.20 \mathrm{~g} / \mathrm{l}$. The patient's initial pelvic radiograph revealed a large right proximal femur osteolytic process with cortical disruption (Fig. 1). Computed tomography (CT) confirmed the presence of a large non-matrix producing soft tissue lesion in the right proximal femur, which was accompanied of cortical destruction, but did not exhibit surrounding sclerosis or associated periosteal reaction (Fig. 2).

Following tumor resection, the patient received bone cement and was subjected to internal fixation. Microscopic examination of the tumor specimen by hematoxylin and eosin stain (ZSGB-BIO, Beijing, China) revealed proliferation of 


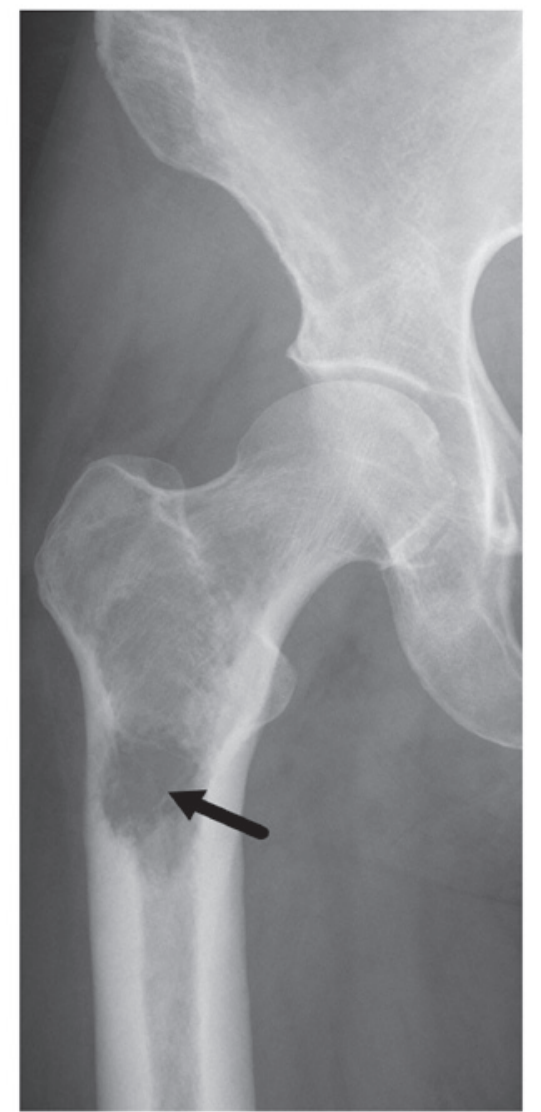

Figure 1. Anteroposterior supine pelvic radiograph revealed a large right proximal femur osteolytic lesion (black arrow)

large polygonal cells separated by thin fibrous septa. The cells presented round nuclei, were disposed centrally, and contained abundant granular eosinophilic cytoplasm. A small number of normal mitoses were also observed (Fig. 3). On immunohistochemistry, the tumor cells were diffusely positive for myoglobin and cytokeratin, focally positive for $\mathrm{Ki}-67$, and negative for cluster of differentiation (CD)138, CD79a, CD20, CD3, CD30, anaplastic lymphoma kinase and chromogranin A (CgA). Consequently, the patient was diagnosed with ASPS of the right proximal femur.

The patient initiated chemotherapy treatment with adriamycin (A) and ifosfamide (I) at 20 days post-surgery. The AI protocol consisted of 4-day administration of ifosfamide at a dose of $3 \mathrm{~g} / \mathrm{m}^{2}$, and 3-day administration of adriamycin at a dose of $60 \mathrm{mg} / \mathrm{m}^{2}$. In addition, the patient received adequate mesna for protection and hydration. The treatment was effective in treating the symptoms, and the patient achieved IV degree of bone marrow suppression. The second round of chemotherapy started three weeks later, and following four weeks, the patient accepted radiotherapy with total doses of $60 \mathrm{~Gy} / 200 \mathrm{cGy} / 30$ fraction/40 day. During this period, the patient complained of persistent pain in the left leg.

The subsequent X-ray examination was normal (Fig. 4), but plain CT scan revealed a focus of marrow replacement, surrounded by a soft tissue mass without cortical bone destruction (Fig. 5). The soft tissue mass displayed homogeneous texture, sharp margins and mild enhancement surrounding the right femoral cava, while the marrow replacement exhibited
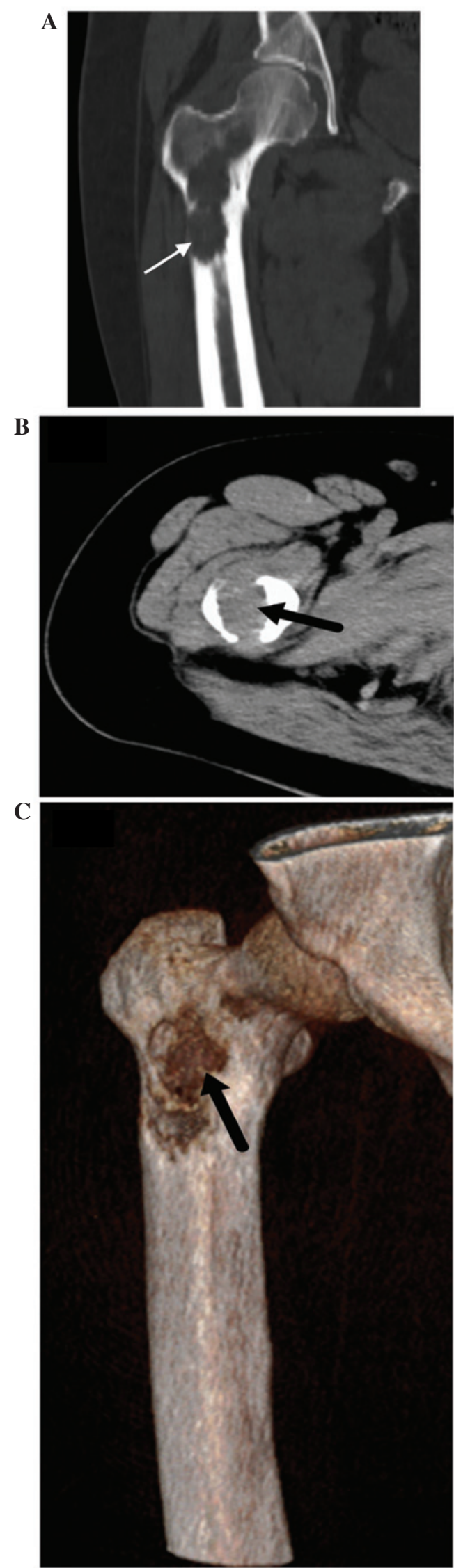

Figure. 2 (A) Coronal multiple planar reconstruction of the femur observed in bone window indicated cortical destruction without internal matrix or associated bone reaction (white arrow). (B) Axial computed tomography of the femur observed in soft tissue window revealed the presence of a soft tissue mass (black arrow). (C) Volume rendering technique detected bone destruction (black arrow). 


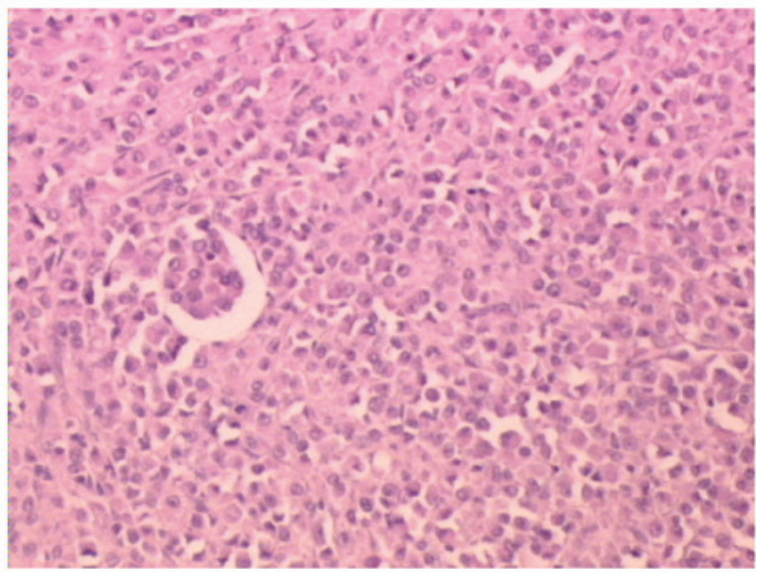

Figure 3. Microscopic examination of the tumor specimen by hematoxylin and eosin stain identified large tumor cells, round or polygonal in shape, with vacuolated cytoplasms abundant in eosinophilic granular bodies (magnification, $\mathrm{x} 100$ ).
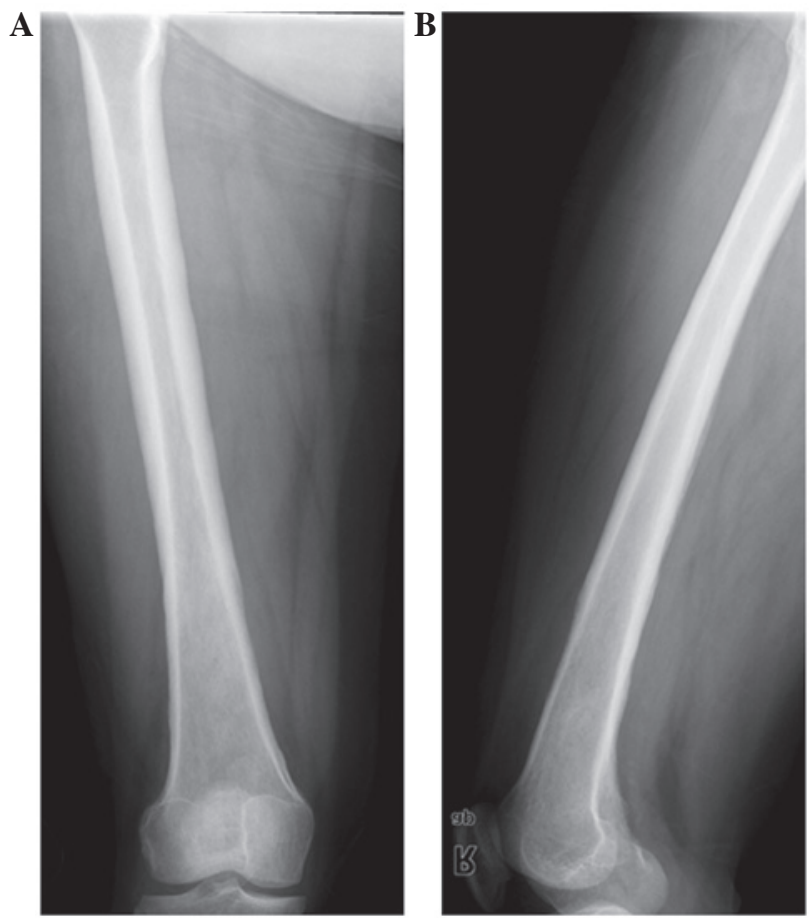

Figure 4. (A) Anteroposterior and (B) lateral radiographs did not reveal any abnormalities in the left femur.

heterogeneous enhancement upon administration of contrast medium (Fig. 6). Magnetic resonance imaging (MRI) demonstrated a clearer lesion extent of marrow replacement, which presented equal signal intensity on T1-weighted imaging and bright signal intensity on T2-weighted imaging. The surrounding soft tissue mass displayed the same signal intensity (Fig. 7).

According to the clinical manifestations and radiological features, second primary tumor of bone rather than metastatic tumor was hypothesized. Following limb salvage treatment, microscopic examination of the specimen revealed that the tumor cells aligned diffusely with remarkable heteromorphism (Fig 8), and the adjacent striated muscles were invaded.

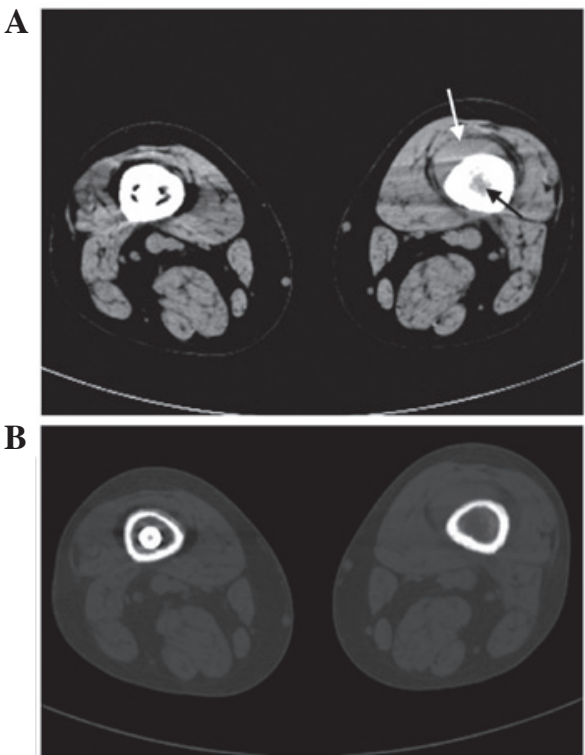

Figure 5. Axial computed tomography. (A) Femur observed in soft tissue window detected the replacement of the normal medullary fat in the left femoral canal by a lesion of soft tissue density (black arrow) and a surrounding soft tissue mass (white arrow). (B) Femur observed in bone window revealed the complete cortical bone.

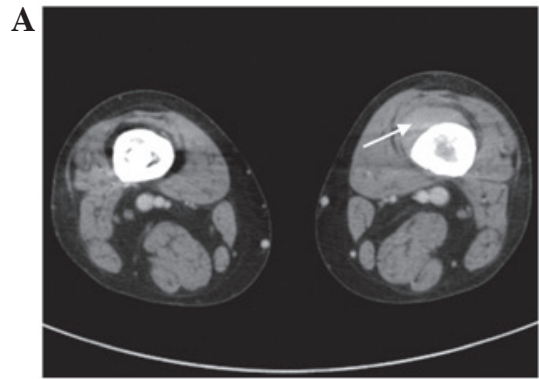

$\mathbf{B}$

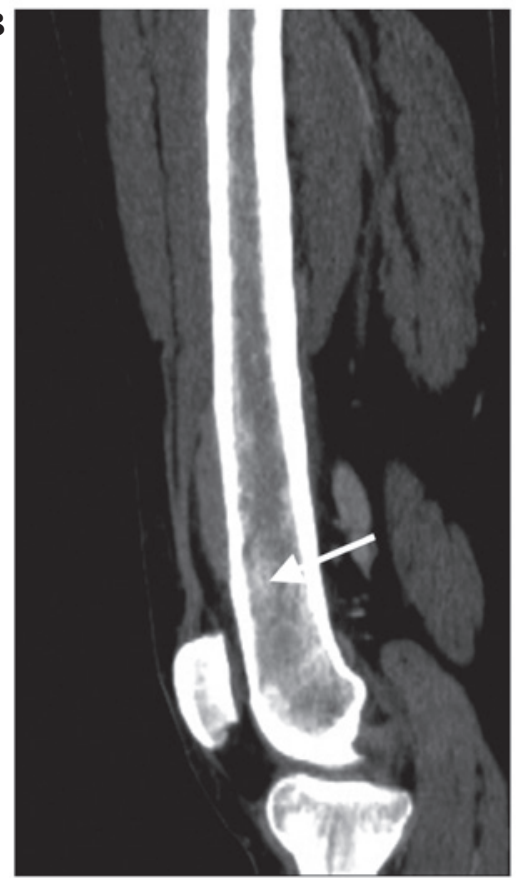

Figure 6. (A) Axial enhanced computed tomography examination of left femur identified an homogeneous and sharply marginated mild enhancement surrounding the right femoral cava (white arrow). (B) Sagittal multiple planar reconstruction demonstrated heterogeneous enhancement in the right femoral canal (white arrow). 
A

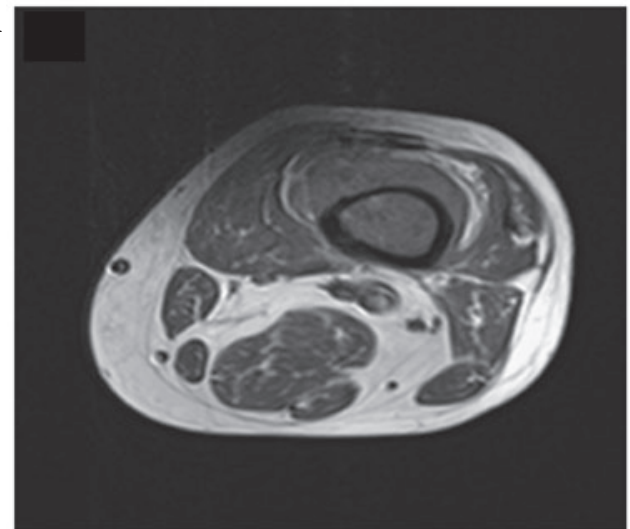

B

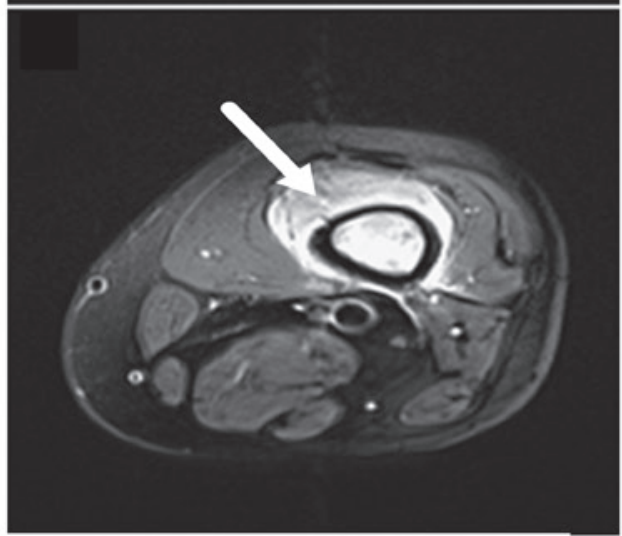

$\mathbf{C}$

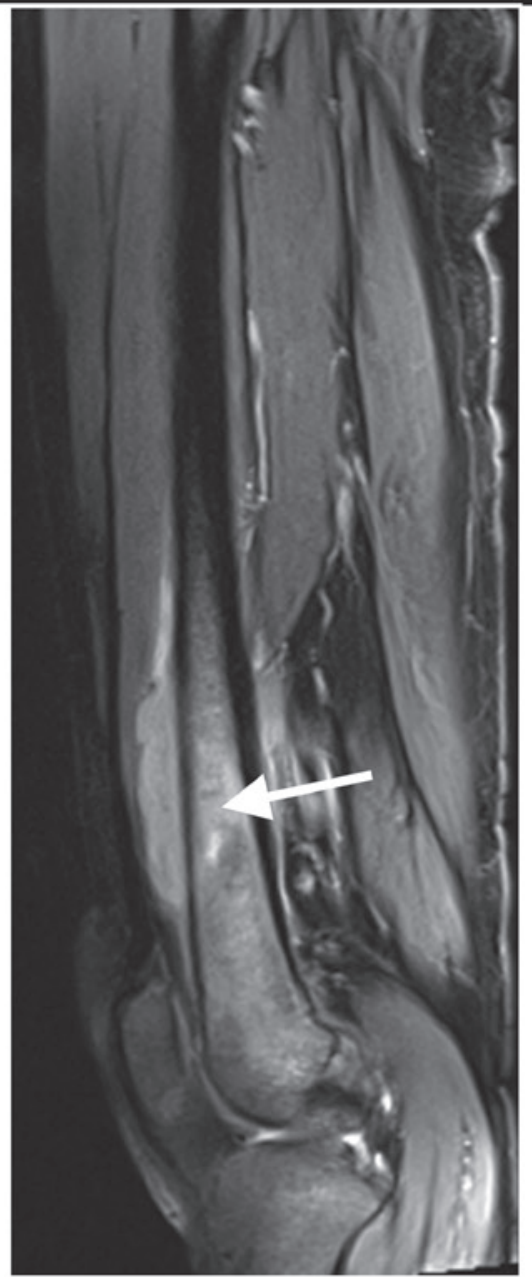

Figure 7. (A) Axial T1-weighted imaging demonstrated the signal intensity of the lesion to be mostly isointense, compared with muscle. (B) Axial and (C) sagittal T2-weighted fat-suppression imaging indicated that the signal intensity of the lesion was high (white arrows).

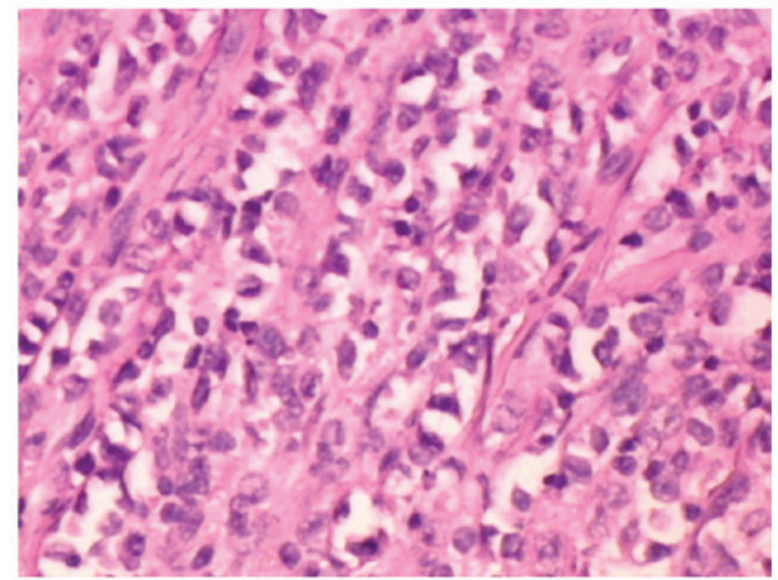

Figure 8. Microscopic examination of the tumor specimen by hematoxylin and eosin stain revealed that the tumor cells aligned diffusely with remarkable heteromorphism (magnification, x200).

Immunohistochemical examination demonstrated the tumor to be leukocyte common antigen ${ }^{+}, \mathrm{CD} 99^{+}$, vimentin-, desmin', melanoma-, synaptophysin', pancytokeratin ${ }^{-}, \mathrm{CgA}^{-}, \mathrm{CD}^{-}$, $\mathrm{CD}_{4}{ }^{+}, \mathrm{CD} 20^{-}$and $\mathrm{CD} 79 \mathrm{a}^{-}$. The pathological diagnosis was established to be peripheral T-cell lymphoma with the invasion of adjacent striated muscles.

According to the clinical manifestation and pathological results, the final diagnosis was ASPS of the right femur and PLB of the left femur. The patient then received radiotherapy, and was followed up for 4 years. To date, there is no evidence of bone marrow aspiration or abnormal laboratory results.

\section{Discussion}

In the present study, the case of a 42-year-old woman diagnosed with ASPS and PLB is reported. The patient underwent en bloc surgical resection, chemotherapy and radiotherapy, and remains currently alive.

The ASPS was located in the right femur, and had invaded the surrounding muscle. This is an unusual location for this tumor in an adult, according to previous reports $(3,5)$. Certain clinical signs and symptoms exhibited by the patient were also considered unusual, such as the results of blood routine test, which suggested anemia.

The microscopic features of tumor cells do not tend to vary from tumor to tumor, and this uniformity is one of the characteristics of ASPS (19). The radiological features of primary bone manifestations of ASPS have been previously described by Park et al (5). A common feature in all the cases of ASPS reported by these authors was the detection of bone destruction with poorly defined tumor margins and epicenter located in the bone, indicating that the tumors occurred primarily in bone. Previous studies have demonstrated that ASPS is distinguishable at equal or slightly increased signal intensity relative to skeletal muscle on T1-weighted MRI, and displays high and heterogeneous signal intensity on T2-weighted MRI. In addition, the administration of intravenous contrast medium results in strong, uniform enhancement of tumor imaging (20). Tumor hypervascularity is characterized by findings such as serpentine flow voids, due to peripheral feeding and 
intratumoral vessels (20). In the present case report, X-ray examination revealed a large right proximal femur osteolytic process with cortical disruption, and CT scan confirmed the presence of a large non-matrix producing soft tissue lesion of the right proximal femur, which exhibited cortical destruction, but no surrounding sclerosis or associated periosteal reaction were observed. Cross-sectional imaging demonstrated that the epicenter was located in the right femur, and the intraosseous soft tissue mass was similar in size to the extraosseous component (19). This evidence supported the hypothesis that the tumor originated in bone.

In conclusion, the clinical symptoms of ASPS experienced by the patient of the present case report were not typical, and imageological diagnosis was the fastest way of confirming the disease, since MRI is the most effective method to determine the dimension of bone destruction and hypervascularity (20). Therefore, ASPS should be considered in the differential diagnosis of bone-originating highly vascularized soft tissue masses.

Currently, there is no consent definition or diagnostic criteria for PLB (10-12,21-25). It has been previously proposed that there may be lymph node involvement if there is a 6-month window between the time of diagnosis of the primary bone focus and the emergence of lymph node disease (21). The diagnosis of PLB may be challenging, since, it is often difficult to differentiate clinically PLB and lymphomatous involvement of bone as a component of extraosseous lymphoma $(22,23)$. The patient of the present case report presented a primary focus in a single bone, which was confirmed histologically. However, there was no evidence of distant lymph node or metastasis to meet the diagnosis criterion. In the present case, the most typical symptom of PLB displayed by the patient was bone pain not relieved by rest, which was insidious and intermittent, similarly to a previous case reported in the literature (21).

PLB is mainly of B-cell origin (10). However, the pathological diagnosis of the patient in the present case report was established to be peripheral T-cell lymphoma of the left femur. Although T-cell PLB has been previously described, its incidence is rare, with the exception of Japan and Hong Kong, where the disease is more common, possibly due to the higher overall incidence of T-cell lymphoma in these countries (24).

The radiological features of PLB are variable and nonspecific (20-22). Conventional radiography typically reveals an osteolytic pattern of bone destruction, but may instead reveal a sclerotic or mixed lytic and sclerotic pattern, or may be normal in $5 \%$ of the cases. In the present case, the results of X-ray examination were normal. Previous studies have reported that periosteal reaction is typically minimal in PLB, despite extreme medullary infiltration (20-22). In addition, the presence of soft tissue masses is common in PLB, but these are better detected by cross-sectional imaging (24), since CT may reveal soft tissue extension, cortical involvement, or marrow invasion suggestive of malignancy, but these findings are nonspecific (20-22). Radionuclide bone scans are abnormal in the majority of patients with PLB (98\%), demonstrating mild to marked increased uptake (22). Positron emission tomography may be aid in staging or detecting residual disease following treatment, but does not contribute to the initial diagnostic examination of suspected PLB (25). In MRI, the characteristic signals of PLB tend to be heterogeneous and variable, and the majority of lesions appear isointense or hypointense compared with muscle on T1, and hypo, iso or hyperintense compared with subcutaneous fat on T2 $(20,23,26)$. The low intensity of the lesion signals on $\mathrm{T} 1$ and $\mathrm{T} 2$ is speculated to be associated with a high content of fibrous tissue in patients with PLB (26). Furthermore, enhancement patterns in PLB are usually heterogeneous (26). Soft tissue extension is present in the majority of cases of PLB, and generally exhibits the same signal intensity than the bone lesion (26). Intramedullary extension is best assessed on MRI, since a clear line of demarcation with normal marrow is usually observed (23). PLB is rarely confined to the periosteum or cortex with diffuse cortical thickening without medullary involvement (23).

With the development of effective oncological treatments, surgery is no longer required in the management of PLB (11). Therefore, an accurate diagnosis is required in order to avoid unnecessary surgical procedures. Biopsy and imaging studies are effective for the diagnosis of PLB, since MRI is the most sensitive technique for the detection of intraosseous tumors (23).

In summary, the case of a 42-year-old woman with SPM has been reported in the present study. To the best of our knowledge, this is the first case of ASPS in the right femur and PLB in the left femur occurring concomitantly in the same patient to date. These two types of tumors present different inherent imageology characteristics. The review of the literature conducted in the present study identified that the incidence of SPMs is not rare (15-18). Therefore, when the radiographic signs are unexpected, the possibility of a second primary tumor should be considered.

\section{References}

1. Weiss SW and Goldblum (eds): Malignant soft tissue tumors of uncertain type. In: Enzinger and Weiss's Soft Tissue Tumors. 5th edition. Mosby Elsevier, Philadelphia, pp1182-1191,2008.

2. Christopherson WM, Foote FW and Stewart FW: Alveolar soft-part sarcomas; structurally characteristic tumors of uncertain histogenesis. Cancer 5: 100-111, 1952.

3. Zhu FP, Lu GM, Zhang LJ, Wang JD, An XJ and Dong YC: Primary alveolar soft part sarcoma of vertebra: A case report and literature review. Skeletal Radiol 38: 825-829, 2009.

4. van Ruth S, van Coevorden F, Peterse JL and Kroon BB: Alveolar soft part sarcoma. A report of 15 cases. Eur J Cancer 38: 1324-1328, 2002

5. Park YK, Unni KK, Kim YW, Han CS, Yang MH, Wenger DE, Sim FH, Lucas DR, Ryan JR, Nadim YA, et al: Primary alveolar soft part sarcoma of bone. Histopathology 35: 411-417, 1999.

6. Yavuz A, Göya C, Bora A and Beyazal M: Primary alveolar soft part sarcoma of the scapula. Case Rep Oncol 6: 356-361, 2013.

7. Zadnik PL, Yurter A, DeLeon R, Molina CA, Groves ML, McCarthy E and Sciubba DM: Alveolar soft-part sarcoma in the sacrum: A case report and review of the literature. Skeletal Radiol 43: 115-120, 2014.

8. Singh T, Satheesh CT, Lakshmaiah KC, Suresh TM, Babu GK, Lokanatha D, Jacob LA and Halkud R: Primary bone lymphoma: A report of two cases and review of the literature. J Cancer Res Ther 6: 296-298, 2010.

9. Oberling C: Les reticulosarcomes et les reticuloendotheliosarcomes de la moelle osseuse (sarcomes d'Ewing). Bull Assoc Fr Etud Cancer 17: 259-296, 1928 (In French).

10. Jawad MU, Schneiderbauer MM, Min ES, Cheung MC, Koniaris LG and Scully SP: Primary lymphoma of bone in adult patients. Cancer 116: 871-879, 2010.

11. Ramadan KM, Shenkier T, Sehn LH, Gascoyne RD and Connors JM: A clinicopathological retrospective study of 131 patients with primary bone lymphoma: A population-based study of successively treated cohorts from the British Columbia Cancer Agency. Ann Oncol 18: 129-135, 2007. 
12. Power DG, McVey GP, Korpanty G, Treacy A, Dervan P, O'Keane C and Carney DN: Primary bone lymphoma: Single institution case series. Ir J Med Sci 177: 247-251, 2008.

13. Lones MA, Sanger W, Perkins SL and Medeiros LJ: Anaplastic large cell lymphoma arising in bone: Report of a case of the monomorphic variant with the $\mathrm{t}(2 ; 5)(\mathrm{p} 23 ; \mathrm{q} 35)$ translocation. Arch Pathol Lab Med 124: 1339-1343, 2000.

14. Dong M, Wei H, Hou JM, Gao S, Yang DZ, Lin ZH, Jia Y, Ren XP and Gao MH: Possible prognostic significance of p53, cyclin D1 and $\mathrm{Ki}-67$ in the second primary malignancy of patients with double primary malignancies. Int J Clin Exp Pathol 7: 3975-3983, 2014.

15. Vaslamatzis M, Alevizopoulos N, Petraki C, Vrionis E, Zoumblios C, Stassinopoulou P, et al: Second primary neoplasms (SPN) in cancer patients. Proc ASCO 22: 3581, 2003.

16. Morgenfeld EL, Tognelli GF, Deza E, Santillan D, Ares S, Morgenfeld E, et al: Synchronous and metachronous second (ST) and third (TT) primary tumors (PT) in a large patient population. Proc ASCO 22: 3152, 2003.

17. Hulikal N, Ray S, Thomas J and Fernandes DJ: Second primary malignant neoplasms: A clinicopathological analysis from a cancer centre in India. Asian Pac J Cancer Prev 13: 6087-6091, 2012.

18. Irimie A, Achimas-Cadariu P, Burz C and Puscas E: Multiple primary malignancies - epidemiological analysis at a single tertiary institution. J Gastrointestin Liver Dis 19: 69-73, 2010.

19. Wakely PE Jr, McDermott JE and Ali SZ: Cytopathology of alveolar soft part sarcoma: A report of 10 cases. Cancer 117: 500-507, 2009.
20. Iwamoto $\mathrm{Y}$, Morimoto N, Chuman H, Shinohara N and Sugioka Y: The role of MR imaging in the diagnosis of alveolar soft part sarcoma: A report of 10 cases. Skeletal Radiol 24: 267-270, 1995.

21. Mulligan ME, McRae GA and Murphey MD: Imaging features of primary lymphoma of bone. AJR Am J Roentgenol 173: 1691-1697, 1999.

22. O’Neill J, Finlay K, Jurriaans E and Friedman L: Radiological manifestations of skeletal lymphoma. Curr Probl Diagn Radiol 38: 228-236, 2009.

23. Heyning FH, Kroon HM, Hogendoorn PC, Taminiau AH and van der Woude HJ: MR imaging characteristics in primary lymphoma of bone with emphasis on non-aggressive appearance. Skeletal Radiol 36: 937-944, 2007.

24. Gill P, Wenger DE and Inwards DJ: Primary lymphomas of bone. Clin Lymphoma Myeloma 6: 140-142, 2005.

25. Takahashi T, Tsukuda H, Itoh H, Kimura H, Yoshimoto $M$ and Tsujisaki M: Primary and isolated adult T-cell leukemia/lymphoma of the bone marrow. Intern Med 50: 2393-2396, 2011.

26. Hermann G, Klein MJ, Abdelwahab IF and Kenan S: MRI appearance of primary non-Hodgkin's lymphoma of bone. Skeletal Radiol 26: 629-632, 1997. 